\title{
Caries Dental su Asociación con el Índice de Masa Corporal en una Población Joven Adulta
}

\author{
Dental Caries its Association with Body Mass Index in a Young Adult Population
}

María del Pilar Adriano Anaya ${ }^{1}$; Tomás Caudillo Joya ${ }^{1}$ \& Pilar Alejandra Caudillo Adriano ${ }^{2}$

\begin{abstract}
ADRIANO, A. M. P.; CAUDILLO, J. T. \& CAUDILLO, A. P. A. Caries dental su asociación con el índice de masa corporal en una población joven adulta. Int. J. Odontostomat., 11(4):437-442, 2017.

RESUMEN: Este estudio tiene como objetivo determinar la distribución del Índice de Masa Corporal y su asociación con la caries dental en estudiantes de primer ingreso período 2016 de la carrera de Cirujano Dentista de la Facultad de Estudios Superiores Zaragoza UNAM. El estudio fue transversal, descriptivo y observacional, en una muestra por conveniencia de 335 estudiantes que entregaron su consentimiento firmado para participar en este estudio. El bajo peso, sobrepeso y obesidad se midió a través del Índice de Masa Corporal (IMC), e interpretado por los criterios establecidos en la Norma Oficial Mexicana NOM-008-SSA3-2010, para el tratamiento integral del sobrepeso y la obesidad y la caries dental por el indicador epidemiológico de Dientes cariados, perdidos y obturados (CPOD). La información se capturó en el Programa Estadístico SPSS versión 17.0. El 3,3\% de esta población presentó bajo peso con un promedio de caries dental de 6,3 \pm 5 . El $54 \%$ normal con un CPOD de 7,6 $\pm 4,8$. El $31 \%$ con sobrepeso y un promedio de caries de 7,0 $0 \pm 5,1$. El $9,3 \%$ con obesidad grado 1 y $7,3 \pm 3,6$ dientes cariado y el $2,4 \%$ con obesidad grado 2 y $5,2 \pm 4,1$ con historia de caries dental. El $31 \%$ de los estudiantes que participaron en este estudio presentaron sobrepeso, por lo que existe un riesgo muy elevado si no se actúa con medidas preventivas y de promoción de la salud, de que estos alumnos en un futuro se conviertan en obesos y con ello tener repercusiones en su salud general.
\end{abstract}

PALABRAS CLAVE: sobrepeso, obesidad, caries dental, IMC.

\section{INTRODUCCIÓN}

El sobrepeso y la obesidad se han convertido en el factor de riesgo modificable más importante en la sociedad mexicana. En la actualidad $71,3 \%$ de los adultos mexicanos padece esta condición, con una prevalencia ligeramente elevada en las mujeres. Por grupo de edad la obesidad es más frecuente en la cuarta y quinta décadas de la vida (Barquera et al., 2013).

Barquera et al. (2006) reportó que en adultos de 20 años o mayores la prevalencia de sobrepeso y obesidad fue de 69,7 \%, lo que ubicó a México como uno de los países con mayor prevalencia en la región de América y a nivel global.

La obesidad es el principal factor de riesgo para el desarrollo de enfermedades crónicas no transmisibles, como la diabetes mellitus y las enfermedades cardiovasculares (las dos principales causas de mor- talidad general en México), entre otras complicaciones (World Health Organization, 2000; World Health Organization/Food and Agriculture Organization, 2003).

El sobrepeso y la obesidad afectan a 7 de cada 10 adultos mexicanos de las distintas regiones, localidades y nivel socio económico. Esto implica que los esfuerzos para prevenir este problema deben tener prioridad nacional al mismo tiempo que se implementan esfuerzos multisectoriales y con participación social para su control. Para ello se requiere regulación, diseño y evaluación de programas y una adecuada inversión de recursos (Encuesta Nacional de Salud y Nutrición, 2012).

Cualquier ciudadano en México, con una dieta común: torta de tamal y champurrado; refrescos para aliviar la sed; guisos en aceite insaturado luego de uso continuo o la famosa dieta "T", a base de Tacos,

${ }^{1}$ FES-Zaragoza UNAM, México.

${ }^{2}$ UAM-Xochimilco, México. 
Tortas, Tamales, Tostadas, Tortillas, Tlacoyos, Tlayudas, Totopos, Tinga y Todo lo que engorde, prácticamente es candidato a contraer diabetes, sobre todo si además de esa mala dieta no hace ejercicio (Galán, 2005).

La dieta rica en hidratos de carbono es uno de los factores de riesgo para el desarrollo de obesidad, así como también para la caries dental donde el problema, no radica sólo en la cantidad que se ingiere, sino también, en la frecuencia con que se consume, el tiempo que permanece en la boca, y los malos hábitos higiénicos que se tengan (Caudillo \& Adriano, 2013; Adriano Anaya et al., 2014).

Puesto que la dieta es un factor determinante en el desarrollo de la caries, es preciso dar una información adecuada a este respecto a los pacientes. Además, no hay que olvidar que un incremento en azúcares no solo supondrá un mayor riesgo de caries sino también un riesgo incrementado a padecer obesidad, y así una mayor predisposición en adultos a sufrir enfermedades como la diabetes, las enfermedades cardiovasculares (hipertensión, colesterol), las respiratorias (apnea, asma), ortopédicas (fracturas) y hepáticas (Palacios et al., 2009; Steyn \& Temple, 2012; González Sanz et al., 2013).

La caries dental es un problema específico del proceso salud enfermedad bucal, que tiene su concreción en la boca con la desmineralización de los dientes (Adriano Anaya et al.). La caries dental, al igual que la obesidad, se encuentra multideterminada por factores económicos, políticos, sociales, biológicos y culturales que los hace complejos en su comprensión y en consecuencia generar propuestas alternativas para su modificación (Galán).

La relación entre caries dental y obesidad no está clara: no se conoce si realmente existe asociación entre ellas o, simplemente, coexisten en el tiempo, ya que comparten etiología común y/o factores contribuyentes similares. Respecto a la asociación positiva directa entre caries e IMC, los estudios (Kelishadi et al., 2010; Pannuzio, 2010; Honne et al., 2012; Adriano et al., 2013), que han encontrado asociación comentan que es debido a que el aumento de peso es por la dieta, sobre todo por la elevada frecuencia de consumo de azúcares y comidas entre horas, que da lugar a un incremento en el número de microorganismos cariogénicos (Adriano et al.). Otra posible explicación, según Modéer et al. (2010), es que los niños obesos presentan menor tasa de saliva total estimulada que los normopesos vs 2,0 $\mathrm{ml} / \mathrm{min}, \mathrm{p}<0,001)$ y una mayor inflamación gingival $(p<0,001)$. Esto concuerda con Pannunzio et al. (2010), quienes afirmaron que niños con mayor IMC presentaron alteraciones en la composición de la saliva (concentración de fosfatos, ácido sialicílico libre, proteínas y actividad de la peroxidasa) como factores favorables para condicionar caries. Sin embargo existen otros estudios (González Martínez et al., 2014), que evidencian que no existe asociación entre ambos problemas.

El determinar la distribución del Índice de Masa Corporal y su asociación con la caries dental en estudiantes de primer ingreso período 2016 de la carrera de Cirujano Dentista de la Facultad de Estudios superiores Zaragoza UNAM nos va a permitir ampliar los marcos explicativos sobre el estudio de éstos dos problemas de Salud pública en la edad de 17 a 32 años, que han sido poco estudiados, de tal manera que permita generar propuestas para modificar y/o solucionar dichos problemas.

\section{MATERIAL Y MÉTODO}

Este Proyecto forma parte de la Línea de Investigación Salud Pública en Estomatología de la Facultad de Estudios Superiores Zaragoza UNAM.

El estudio fue transversal, descriptivo y observacional realizado en una muestra por conveniencia de 335 estudiantes de primer ingreso período 2016 de la carrera de Cirujano Dentista, conformada por los que entregaron su permiso firmado previa información de los objetivos del proyecto.

La exploración de la cavidad oral la realizaron seis pasantes previamente capacitados y calibrados (KAPPA Intra e interexaminadores $\mathrm{K}=89 \%$ ) en la obtención del Índice CPOD, $(\mathrm{C}=$ cariados, $\mathrm{P}=$ perdidos, $\mathrm{O}=$ obturados $\mathrm{D}=$ diente), siguiendo las recomendaciones de la OMS (1997). Este indicador es recomendado por la organización Mundial de la Salud debido a que es mundialmente confiable, estandarizado y permite la comparación con otros estudios (Adriano et al.).

Se estandarizaron también en el manejo de la antropometría (peso, talla, Índice de Masa Corporal), siguiendo el método de Habitch (Habitch et al., 1974; Habitch et al., 1974; de Onis \& Habicht, 1996). Se pesó a los escolares con una báscula digital de cristal templado de 182 kilos de capacidad y la talla se llevó a cabo con un estadiómetro marca SECA de $200 \mathrm{CM}$. Tomando a esta como la distancia que media entre la parte más alta de la cabeza y la planta de los pies. 
Se utilizaron las barreras de protección planteadas por la Norma Oficial Mexicana para el control y manejo de infecciones bucales, (NOM-087-ECOLSSA1., 2000; NOM-087-ECOL-SSA1., 2002).

Una vez pesados y medidos los escolares se obtenía el índice de masa corporal IMC, que se define como una medida de asociación entre el peso y la talla de un individuo. Ideado por el estadístico belga L. A. J. Quetelet, por lo que también se conoce como índice de Quetelet, (Rodríguez et al., 2010).
La clasificación para la interpretación del IMC fueron los propuestos por los criterios establecidos en la Norma Oficial Mexicana NOM-008-SSA3 (2010) Tabla I, y NOM-043-SSA2 (2012) para el tratamiento integral del sobrepeso y la obesidad.

La información se capturó en el Programa de cómputo SPSS versión 17.0 se obtuvieron: Estadísticas descriptivas como es la media, de caries dental e higiene oral, \% de IMC, Anova (Bonferroni), para IMC y caries dental, t de Student para significancia de caries dental y sexo y X2 de Pearson para significancia entre caries dental y edad.

Tabla I. Norma Oficial Mexicana Nom-008-Ssa3-2010, para el Tratamiento Integraldel Sobrepeso y la Obesidad.

\begin{tabular}{lll}
\hline & \multicolumn{2}{c}{ TRATAMIENTO INTEGRAL DEL SOBREPESO } \\
Y LA OBESIDAD
\end{tabular}

\section{RESULTADOS}

El total de estudiantes que participaron en este estudio fue de 335 , las edades tuvieron un rango de 17 a 32 años de edad. Por el número de estudiantes de 22 a 32 años se agruparon en un solo rango. A los 18 años es donde se agrupó el mayor número de estudiantes (155). El promedio general de caries dental para este grupo de población fue de 7,3 dientes con historia de la enfermedad, si tomamos en cuenta los 28 dientes que hay en la boca después de los 12 años de edad, podemos observar que la tercera parte de los dientes permanentes se encuentran dañados por caries dental. Se encontró significanciaestadística entre la edad y caries dental. Tabla II.

Con respecto a la distribución de los estudiantes y el Índice de Masa Corporal, la prevalencia de sobrepeso y obesidad fue del 43 $\%$, con bajo peso del $3 \%$ y normalidad del 54 $\%$.

Tabla III. Índice de Masa Corporal por edad y promedio de caries dental de los estudiantes Índice de Masa Corporal.

\begin{tabular}{ccccccc}
\hline Edad & Bajo peso & Normal & Sobrepeso & Obeso 1 & Obeso 2 & Total \\
\hline 17 & 1 & 16 & 8 & 2 & 1 & 28 \\
18 & 3 & 87 & 52 & 11 & 2 & 155 \\
19 & 3 & 43 & 21 & 7 & 3 & 77 \\
20 & 2 & 18 & 15 & 4 & 1 & 40 \\
21 & 1 & 10 & 1 & 4 & 0 & 16 \\
$22-32$ & 1 & 7 & 7 & 3 & 1 & 19 \\
Total & 11 & 181 & 104 & 31 & 8 & 335 \\
$\%$ & 3,3 & 54 & 31 & 9,3 & 2,4 & $100,0 \%$ \\
CPOD & $6,3 \pm 5,1$ & $7,6 \pm 4,8$ & $7,0 \pm 5,1$ & $7,3 \pm 3,6$ & $5,2 \pm 4,1$ & $7,3 \pm 4,8$ \\
\hline \multicolumn{1}{r}{} & & & & & \multicolumn{3}{c}{ ANOVA $\mathrm{p}=1,000$} \\
\hline
\end{tabular}


ADRIANO, A. M. P.; CAUDILLO, J. T. \& CAUDILLO, A. P. A. Caries dental su asociación con el índice de masa corporal en una población joven adulta. Int. J. Odontostomat., 11(4):437-442, 2017.

Tabla IV. Distribución del Índice de Masa Corporal por sexo y promedio CPOD de los estudiantes.

\begin{tabular}{|c|c|c|c|c|c|c|c|}
\hline \multirow[b]{2}{*}{ Sexo } & \multicolumn{5}{|c|}{ Índice de Masa Corporal } & \multirow[b]{2}{*}{ Total } & \multirow[b]{2}{*}{ CPOD } \\
\hline & Bajo peso & Normal & Sobrepeso & Obeso 1 & Obeso 2 & & \\
\hline Masculino & 3 & 51 & 34 & 9 & 4 & 101 & $6,7 \pm 4,6$ \\
\hline Femenino & 8 & 130 & 70 & 22 & 4 & 234 & $7,5 \pm 4,8$ \\
\hline Total & 11 & 181 & 104 & 31 & 8 & 335 & $\begin{array}{r}7,3 \pm 4,8 \\
p=0,252\end{array}$ \\
\hline
\end{tabular}

El promedio de caries dental más elevado fueron los que cayeron en el rubro normalidad con 7.6 dientes con historia de la enfermedad. Al realizar comparaciones múltiples entre la edad, el IMC y los promedios de caries dental la prueba ANOVA no reportó ninguna significancia Estadística (Tabla III).

Del total de los estudiantes 101 (30\%), fueron del sexo masculino y 234 (70 \%) del femenino.

Los promedios de caries dental con respecto al sexo fueron similares no encontrando diferencias estadísticas $(p=0,252)$.

Al realizar múltiples comparaciones entre el Îndice de Masa Corporal, el sexo y los promedios de caries dental no se encontró ninguna asociación entre ellos $(p=1,000)$ (Tabla IV).

De los 104 estudiantes con sobrepeso 34 son masculino (33\%), y 70 del femenino (67\%). Con respecto a los obesos el $29 \%$ es el sexo masculino (9), y $71 \%$ del femenino ( $N=22)$. Los obesos se comportaron con el mismo número de estudiantes.

El $27 \%$ de los masculinos y $73 \%$ de los del sexo femenino se encontraron con bajo peso.

Esta situación puede deberse a que en esta población participaron más mujeres que hombres (Tabla IV).

\section{DISCUSIÓN}

Determinar la distribución del Índice de Masa Corporal y su asociación con la caries dental en estudiantes de primer ingreso período 2016 de la carrera de Cirujano Dentista de la Facultad de Estudios Superiores Zaragoza UNAM, nos va a permitir conocer la prevalencia de bajo peso, sobrepeso, obesidad, y caries dental de nuestros alumnos que están ingresando a estudiar dicha carrera para implementar las medidas necesarias a partir de programas, de protección específica, promoción de la salud bucal, de curación y rehabilitación, y con ello en el transcurso de cuatro años lograr una población estudiantil con cero caries dental y el sobrepeso y obesidad controlados.

La prevalencia de estudiantes con sobrepeso y obesidad fue del $43 \%$, con bajo peso del $3 \%$ y normalidad del $54 \%$. Sin embargo sólo la de sobrepeso es del $31 \%$ muy por arriba, de lo reportado en la Encuesta Nacional de Nutrición 1999, que a nivel nacional reporta una prevalencia del 19,5\%, por otra parte, el dato reportado a nivel de la Ciudad de México fue del 26,6 \% (Zelocuatecatl et al., 2005). A pesar de que estos estudiantes están iniciando una carrera dentro de las Ciencias de la Salud, se observa un promedio de caries dental de 7.3 dientes con historia de la enfermedad y un porcentaje de sobrepeso y obesidad muy elevado (43\%), lo que indica que tienen una cultura de la enfermedad, por lo que hay que actuar con programas de prevención y promoción de la salud, para generar en ellos una transformación hacia una cultura de la salud.

Se considera que el sobrepeso y la obesidad, es más frecuente en el sexo femenino que en el masculino. En los resultados que estamos presentando encontramos a 143 mujeres con sobrepeso y obesidad (435), y a 47 hombres (14\%), sin embargo en este estudio el $70 \%$ de las participantes fueron mujeres.

Hasta la fecha, no se conoce si realmente existe una asociación entre la caries dental y la obesidad, Pannunzio et al. afirmo que niños con mayor IMC, presentaron alteraciones en la composición de la saliva (concentración de fosfatos, ácido salicílico libre, proteínas y actividad de la peroxidasa) los que son considerados como factores favorables para condicionar caries. Por otro lado la alta ingesta de carbohidratos, su permanencia en la boca y la falta del cepillado dental es otra de las causas de la obe- 
sidad y de la caries dental. Sin embargo los estudios que se han realizado, muestran resultados heterogéneos ya que algunos han encontrado esta asociación y otros no, por lo que es necesario seguir investigando sobre este tema.

\section{CONCLUSIONES}

El $31 \%$ de los estudiantes que participaron en este estudio presentaron sobrepeso, por lo que existe un riesgo muy elevado si no se actúa con medidas preventivas y de promoción de la salud, de que estos alumnos en un futuro se conviertan en obesos y con ello tener repercusiones en su salud general.

Los resultados encontrados en el estudio, no se observó una asociación entre el índice de masa corporal y la caries dental en los estudiantes de primer ingreso período 2016 de la carrera de Cirujano Dentista de la FES-Zaragoza.

ADRIANO, A. M. P.; CAUDILLO, J. T. ; CAUDILLO, A. P. A. Dental caries its association with Body Mass Index in a young adult population. Int. J. Odontostomat., 11(4):437-442, 2017.

ABSTRACT: The objective of this study is to determine the body mass index and its association with dental caries, in first-year students of the dental surgeon degree program for the 2016 school term, Zaragoza College for Higher Education, UNAM. This was a cross, descriptive and observational study, in a sample of 335 students. Low weight, overweight and obesity were measured through body mass index and interpreted with the official Mexican Norm NOM008-SSA3-2010, for integral treatment of overweight, obesity and dental caries. The measures were taken according to the epidemiologic indicator of decayed, lost and sealed teeth (dlst). We used statistical program SPSS version 17.0 for the information. In the studied population, $3.3 \%$ presented low weight, with an average of dental caries of $6.3 \pm 5 ; 54 \%$ presented normal weight with a DLST of $7.6 \pm 4.8 ; 31 \%$ presented with overweight and an average of caries of $7.0 \pm 5.1 ; 9.3 \%$ with obesity grade 1 and $7.3 \pm 3.6$ tooth decay and lastly $2.4 \%$ with obesity grade 2 and $5.2 \pm 4.1$ with a history of dental caries. Of the students participating in this study, $31 \%$ presented overweight, indicating a high risk of poor oral health. If preventive measures in their overall health are not implemented early on, these students are at risk of obesity and general health repercussions in the future.

BMI.

\section{REFERENCIAS BIBLIOGRÁFICAS}

Adriano Anaya, M. P.; Caudillo Joya, T.; Juárez López, M. L. A. \& Caudillo Adriano, P. A. Obesity and dental caries public health problems in a school population. Int. J. Odontostomatol., 8(3):475-80, 2014.

Adriano, P.; Caudillo, T. \& Gómez, A. Epidemiologia Estomatológica. $2^{a}$ ed. Ciudad de México, Facultad de Estudios Superiores Zaragoza, UNAM, 2013. pp.59-64.

Barquera, S.; Campos-Nonato, I.; Hernández-Barrera, L.; Flores, M.; Durazo-Arvizu, R.; Kanter, R. \& Rivera, J. A. Obesity and central adiposity in Mexican adults: results from the Mexican National Health and Nutrition Survey 2006. Salud Publica Mex., 51 Suppl. 4:S595-603, 2006.

Barquera, S.; Campos-Nonato, I.; Hernández-Barrera, L.; Pedroza-Tobías, A. \& Rivera-Dommarco, J. A. Prevalencia de obesidad en adultos mexicanos, ENSANUT 2012. Salud Publica Mex., 55(2):S151-60, 2013.

Caudillo, T. \& Adriano, P. Desnutrición, Obesidad y Caries Dental. Problemas de Salud Púlica en Población Escolar. Ciudad de México, Facultad de Estudios Superiores Zaragoza, UNAM, 2013. pp.67-77.

de Onis, M. \& Habicht, J. P. Anthropometric reference data for international use: recommendations from a World Health Organization Expert Committee. Am. J. Clin. Nutr., 64(4):650-8, 1996.

Encuesta Nacional de Salud y Nutrición 2012. Evidencia para la Política Pública en Salud. Ciudad de México, Instituto Nacional de Salud Pública, 2012. Disponible en: http:// ensanut.insp. $\mathrm{mx} /$ doctos/analiticos/ObesidadAdultos.pdf.

Galán, J. La dieta común del mexicano lo lleva a padecer enfermedades como la diabetes. Noticia. Ciudad de México, La Jornada, 2005. Disponible en: http:// w w w. jornada.unam.mx/2005/10/01/ index.php?section=sociedad\&article $=041 \mathrm{n} 1 \mathrm{soc}$

González Martínez, F. D.; Vidal Madera Anaya, M. \& Tirado Amador, L. R. Relación entre obesidad y caries dental en niños. Rev. Cuba. Estomatol., 51(1):93-106, 2014.

González Sanz, A. M.; González Nieto, B. A. \& González Nieto, E. Salud dental: relación entre la caries dental y el consumo de alimentos. Nutr. Hosp., 28 Suppl. 4:64-71, 2013.

Habicht, J. P.; Martorell, R.; Yarbrough, C.; Malina, R. M. \& Klein, R. E. Height and weight standards for preschool children. How relevant are ethnic differences in growth potential? Lancet, 1(7858):611-4, 1974.

Habitch, J. P. Estandarización de métodos epidemiológicos cuantitativos sobre el terreno. Bol. Sanit. Panam., 76(5):375-84, 1974.

Honne, T.; Pentapati, K.; Kumar, N. \& Acharya, S. Relationship between obesity/overweight status, sugar consumption and dental caries among adolescents in South India. Int. J. Dent. Hyg., 10(4):240-4, 2012. http:// dof.gob.mx/nota_detalle. php?codigo $=5285372 \&$

Kelishadi, R.; Mortazavi, S.; Hossein, T. R. \& Poursafa, P. Association of cardiometabolic risk factors and dental caries in a population-based sample of youths. Diabetol. Metab. Syndr., 2:22, 2010. 
ADRIANO, A. M. P.; CAUDILLO, J. T. \& CAUDILLO, A. P. A. Caries dental su asociación con el índice de masa corporal en una población joven adulta. Int. J. Odontostomat., 11(4):437-442, 2017

Modéer, T.; Blomberg, C. C.; Wondimu, B.; Julihn, A. \& Marcus, C. Association between obesity, flow rate of whole saliva, and dental caries in adolescents. Obesity (Silver Spring), 18(12):2367-73, 2010.

NORMA Oficial Mexicana NOM-008-SSA3-2010, para el tratamiento integral del sobrepeso y la obesidad. Ciudad de México, Secretaría de Salud, 2010. Disponible: http:// www.dof.gob.mx/nota_detalle.php?codigo=5154226\&

NORMA Oficial Mexicana NOM-043-SSA2-2012, Servicios básicos de salud. Promoción y educación para la salud en materia alimentaria. Criterios para brindar orientación. Ciudad de México, Secretaría de Salud, 2012. Disponible en:

NORMA Oficial Mexicana NOM-087-ECOL-SSA1-2002, Protección ambiental - Salud ambiental - Residuos peligrosos biológico-infecciosos - Clasificación y especificaciones de manejo. Ciudad de México, Secretaría de Salud, 2003. Disponible en: http://www.salud.gob.mx/unidades/ cdi/nom/087ecolssa.html

Organización Mundial de la Salud (OMS). Encuestas de Salud Bucodental. Métodos Básicos. Ginebra, Organización Mundial de la Salud, 1997. pp.33-7.

Palacios, C.; Joshipura, K. \& Willett, W. Nutrition and health: guidelines for dental practitioners. Oral Dis., 15(6):36981, 2009.

Pannunzio, E.; Amancio, O. M.; Vitalle, M. S.; Souza, D. N.; Mendes, F. M. \& Nicolau, J. Analysis of the stimulated whole saliva in overweight and obese school children. Rev. Assoc. Med. Bras. (1992), 56(1):32-6, 2010.

Rodríguez, G.; Moreno, L. \& Sarría, A. La obesidad en la Historia. Sobre el índice de Quetelet y obesidad. Rev. Esp. Obes., 8:34-40, 2010.

Steyn, N. P. \& Temple, N. J. Evidence to support a foodbased dietary guideline on sugar consumption in South Africa. BMC Public Health, 12:502, 2012.

World Health Organization (WHO). Obesity: Preventing and Managing the Global Epidemic. Report of a WHO Consultation. Technical Report Series No. 894. Geneva, World Health Organization, 2000.

World Health Organization/Food and Agriculture Organization. Diet, Nutrition and the Prevention of Chronic Diseases. Report of a Joint WHO/ FAO Expert Consultation. WHO Technical Report Series 916. Geneva, World Health Organization, 2003.

Zelocuatecatl, A. A.; Ortega, M. M. \& Fuente, H. J. Asociación entre el índice de masa corporal y las condiciones bucales en escolares. Rev. Odontol. Mex., 9(4):185-90, 2005.
Dirección para correspondencia

Maria del Pilar Adriano Anaya

FES-Zaragoza UNAM

Batalla 5 de Mayo SN

Iztapalapa, Ejercito Oriente, 09230

Ciudad de México

MÉXICO

Email: adriano124@hotmail.com

Recibido : 08-08-2017

Aceptado: 13-10-2017 